years. The cause of a low absolute neutrophil count (ANC) was autoimmune neutropenia in 37 patients, 31 had chronic idiopathic neutropenia, 27 had infectious neutropenia, 3 had cyclic neutropenia, while 2 were diagnosed with alloimmune neonatal neutropenia. The mean value of ANC at presentation was $732 / \mathrm{mm} 3$, and the mean lowest ANC detected during disease course was $600 / \mathrm{mm} 3$. Among the 5 diagnostic subgroups, both values were the lowest in patients with cyclic neutropenia in which mean ANC at presentation was $420 / \mathrm{mm} 3$ and mean lowest ANC detected during disease course was 180/ $\mathrm{mm} 3$.

Granulocyte colony-stimulating factor (GCS-F) was given to only 3 patients, whose mean ANC at presentation was 140/ $\mathrm{mm} 3$. Besides the laboratory finding of persistent severe neutropenia, all 3 patients were prone to recurrent infections. Twenty-three patients had additional cytopenia, out of which 4 had pancytopenia, 13 had anemia and 6 patients had thrombocytopenia. Among patients with additional cytopenia, 5 had positive anti-granulocyte antibodies (21.7\%). The mean time to disease resolution was 10.2 months, being the longest in the cyclic neutropenia subgroup, while patients with infectious neutropenia mainly recovered after 21.3 days. The mean follow-up time was 1.6 years.

According to data from our Department, neutropenia is most commonly diagnosed in pre-school children, boys being more frequently affected than girls. More than one third of patients have positive anti-granulocyte antibodies. However, the condition is usually benign, it resolves mainly spontaneously in less than a year, and patients generally do not require G-CSF.

\section{IMMUNOTHERAPY IN HIGH RISK NEUROBLASTOMA - TREATMENT ALGORITHM OF COMPLICATIONS}

Jasminka Stepan-Giljevic*, Gordana Jakovljevic, Aleksandara Bonevski, Filip Jadrijevic Cvrlje, Nada Rajacic, Maja Pavlovic, Izabela Kranjcec. Department of hematology and oncology „Dr Mladen Cepulic', Children's Hospital Zagreb

\subsection{6/archdischild-2021-europaediatrics.300}

We report our center experience in immunotherapy of high risk neuroblastoma, in particular concerning the most common side effects and their management.

Multidisciplinary team approach will be discussed as well, as it is especially important in care for these patients.

Retrospective review of hospital medical data basis High risk neuroblastoma patients receive intensive multimodal therapy that includes induction, consolidation, and postconsolidation phases. The postconsolidation or maintenance phase implies immunotherapy with anti-GD2 monoclonal antibody dinutuximab beta, applied with the purpose of eliminating any residual tumor cells that may exist after induction and consolidation treatment (multiagent chemotherapy, surgery, high-dose chemotherapy and autologous stem cell transplant).

During the 3 -year period $(2017$ - 2019) 8 high risk neuroblastoma patients received immunotherapy with dinutixumab beta in our center. Patients were in remission confirmed by different methods of investigation. Dinutuximab beta was applied in hospital conditions, as a 10-day continuous infusion, with premedication and concomitant medications that included crystalloid fluids, various pain medications, antihistamines, antiemetics and antipyretics, requiring double lumen central venous catheter and continuous monitoring of vital functions.

Most of our patients achieved adequate pain control with gabapentin and titrated doses of opioids. Fever was a common side effect easily managed by NSAIDs. In case of allergic reaction the rate of dinutuximab beta was decreased by half and restored to its full rate after resolution of symptoms. In the meantime additional doses of antihistamines, oxygen, salbutamol and racemic epinephrine were applied depending on the clinical condition.

Special care was dedicated to fluid balance supervision due to the risk of capillary leak syndrome. Although 5 of our patients had some degree of capillary leak, only one patient developed significant hypotension which was recognized and treated in timely manner with fluid boluses and epinephrine titrated to effect. Upon resolution of hypotension, the drug infusion was restarted at 50\% rate during 2 hours and after that in the absence of recurrent hypotension increased to the full rate.

Postconsolidation immunotherapy with dinutuximab beta has become the standard of care for high-risk neuroblastoma patients. The benefits of this kind of therapy do not come without risks. Having front-line providers who are familiar with immunotherapy and its associated toxicities is critical to the safe and consistent administration of this complex therapy. The team includes physicians of different specialities, nurse practitioners, physician assistants and bedside nurses.

\section{DIAGNOSTICS AND TREATMENT OF CHILDHOOD MALIGNANT LIVER TUMORS - A SINGLE CENTER EXPERIENCE}

Jasminka Stepan-Giljevic*, Domagoj Buljan, Aleksandara Bonevski, Nada Rajacic, Gordana Jakovljevic, Filip Jadrijevic Cvrlje, Maja Pavlovic, Izabela Kranjcec. Department of hematology and oncology „Dr Mladen Cepulic', Children's Hospital Zagreb

\subsection{6/archdischild-2021-europaediatrics.301}

We report our center experience in medical management of malignant childhood liver tumors Retrospective review of hospital medical data basis In the period of 19 years 13 patients were diagnosed and treated in our institution due to malignant liver tumor (8 males and 5 females). Average age at diagnosis was 4,04 years. 3 of them were born prematurely.

Histopathological type of the tumor corresponded to hepatoblastoma in all cases, except one which is the case of hepatocellular carcinoma. The most frequent PRETEXT group was stage 2. Radiological imagaing and biopsy were important in making the correct diagnosis. The usual medical management consisted of neoadjuvant chemotherapy and surgical procedure followed by adjuvant chemotherapy. Overall survival is $76,9 \%$ and the average follow-up period 71,1 months.

Malignant childhood liver tumors are challenging to manage necessitating multidisciplinary approach and collaboration.

\section{4-MONTH-OLD BOY WITH REFRACTORY THROMBOCYTOPENIA AND WISKOTT-ALDRICH SYNDROME - CASE REPORT}

Agnieszka Słowińska*, Karolina Bohdankiewicz, Paulina Dziuba. Wojewódzki Specjalistyczny Szpital Dziecięcy w Olsztynie

10.1136/archdischild-2021-europaediatrics.302 